\title{
AUTOAMOSTRADOR DE BAIXO CUSTO PARA ANÁLISE POR INJEÇÃO EM FLUXO
}

\author{
Marcos Y. Kamogawa* \\ Departamento de Ciências Exatas, Escola Superior de Agricultura “Luiz de Queiroz”, Universidade de São Paulo, CP 9, \\ 13418-900 Piracicaba - SP, Brasil \\ Marcelo Alves Teixeira \\ Universidade Estadual de Mato Grosso do Sul, Rod. Dourados Itahun, km12, 79830-000 Dourados - MS, Brasil
}

Recebido em 24/7/08; aceito em 4/2/09; publicado na web em 3/7/09

\begin{abstract}
A LOW COST AUTOSAMPLER FOR FLOW INJECTION ANALYSIS. This paper describes a homemade autosampler for flow injection analysis (FIA), constructed with two step motors from old floppy disk drives (51/4-inch). The autosampler was connected to a computer through the parallel port and the sampling process was controlled by software in Quick Basic. The performance of the system was assessed by the determination of ammonium, based on the gaseous diffusion into a bromocresol purple solution, following the spectrophotometric determination of change in absorbance. The easy and simple construction is the main characteristics of this equipment and analytical results with RSD lower than $1 \%$ were obtained.
\end{abstract}

Keywords: ammonium; parallel port; step motors.

\section{INTRODUÇÃO}

A análise por injeção em fluxo (FIA), proposta na metade dos anos 70 representou um avanço significativo no campo da automação em química analítica, ${ }^{1}$ contribuindo sensivelmente na evolução dos processos de introdução de amostras, sistemas de detecção e propulsão. ${ }^{2,3}$ A facilidade em se controlar o tempo do procedimento de injeção a partir de dispositivos eletrônicos e com a construção/acoplamento de detectores controlados por microcomputador, permitiu que os sistemas assumissem características automáticas. ${ }^{4}$

Segundo Pasquini e Faria, ${ }^{4}$ a automação dos sistemas em fluxo é possível em quatro pontos: substituição automática da amostra normalmente executada com auxílio de um amostrador automático ou autoamostrador; introdução da amostra no sistema - podem ser empregadas válvulas solenóides, sistemas mecânicos controlados eletronicamente etc.; controle do detector e, interface de aquisição dos dados. Para que um sistema seja automático, os dados devem ser armazenados e processados por uma rotina programada, retornando uma decisão operacional.

O uso de dispositivos eletrônicos em sistemas FIA tem se destacado na mecanização da introdução da amostra, no controle do detector e na aquisição dos dados. Entretanto, a troca das amostras frequentemente é realizada de forma manual pelo operador, o que permite a associação de erros na injeção e uma diminuição da frequência das análises. ${ }^{5} \mathrm{O}$ uso de um autoamostrador acoplado aos módulos de análise permite a execução do procedimento na ausência do operador, tornando estes sistemas atrativos para rotinas laboratoriais.

Figueiredo e colaboradores ${ }^{5}$ empregando peças recuperadas de um equipamento de absorção atômica antigo propuseram a construção de um autoamostrador - injetor comutador para a determinação de fósforo em águas. O sistema controlado por uma interface comercial (PCL711, Advantech) realizou com sucesso o procedimento, demonstrando robustez e facilidade de operação. Diferentes modelos e configurações de autoamostradores estão disponíveis comercialmente, entretanto a um custo relativamente alto quando comparado aos sistemas FIA usuais.

*e-mail: kamogawa@esalq.usp.br
Visando a construção de um sistema de baixo custo, o objetivo deste trabalho foi apresentar uma configuração simples de autoamostrador para análise em fluxo, construído a partir do aproveitamento de motores de passo encontrados em leitores antigos de disquetes (51/4), controlados a partir da porta paralela do microcomputador, utilizando como drive de potência o circuito integrado ULN2803AG (Toshiba).

\section{PARTE EXPERIMENTAL}

\section{Descrição do autoamostrador}

O autoamostrador foi construído empregando-se componentes de duas antigas unidades leitoras de disquete de $51 \frac{1}{4}$ polegadas. $\mathrm{Na}$ montagem utilizaram-se dois motores de passo, uma placa de fibra de madeira de média densidade (MDF), uma haste fina de metal e parafusos de fixação. Na placa de MDF $(25 \mathrm{~cm}$ de raio e $1 \mathrm{~cm}$ espessura) foram efetuados 50 furos equidistantes entre si, com 2 $\mathrm{cm}$ de diâmetro, a um raio de $22,5 \mathrm{~cm}$ do ponto central. Os motores de passo, a haste fina de metal, o MDF e os parafusos de fixação foram unidos conforme descrição apresentada na Figura 1.

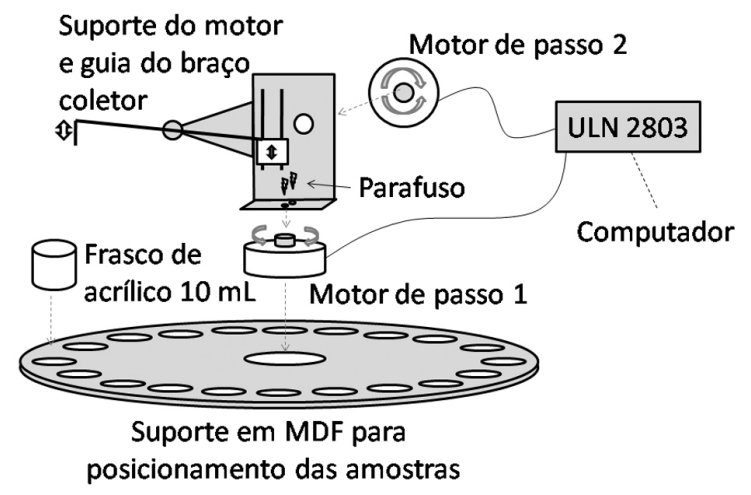

Figura 1. Componentes e esquema de montagem do amostrador automático 
O motor de passo posicionado horizontalmente (motor 1), fixado na base da estrutura de MDF, tem como função definir a posição do braço de amostragem no plano xy, com rotação de $3,6^{\circ}$ por passo, sobre os frascos das amostras, com liberdade de giro de $360^{\circ}$. O outro motor de passo (motor 2), utilizado para a movimentação do braço de amostragem no plano xz, foi mantido em sua estrutura original, com as guias de locomoção do leitor de disquete.

Com o acionamento do motor 2, a guia pode ser deslocada para cima ou para baixo (movimento vertical) em um percurso definido e limitado. Neste percurso, uma haste metálica fixada na estrutura das guias de locomoção permite que o capilar de amostragem seja introduzido no frasco contendo a solução de interesse $(10 \mathrm{~mL})$. Este conjunto fixado diretamente no eixo do motor 1 torna possível a soma dos componentes de movimento na horizontal (motor 1) e vertical (motor 2) do capilar de amostragem, possibilitando que o capilar seja introduzido nos frascos das soluções de referência e das amostras, sequencialmente.

O controle dos motores de passo foi realizado por uma interface de potência utilizando o circuito integrado (ULN2803AG) ligado à porta paralela do microcomputador, como descrito por RódenasTorralba $^{6}$ e Messias. ${ }^{7}$

A configuração apresentada possui a finalidade de compatibilizar a intensidade de corrente e a diferença de potencial necessária para o acionamento dos motores de passo, porque os motores de passo consomem cerca de 0,2 A de corrente em uma diferença de potencial de $12 \mathrm{~V}$. Por outro lado, a porta paralela do microcomputador fornece, apenas, uma intensidade de corrente em torno de $2 \mathrm{~mA}$ e diferença de potencial de $5 \mathrm{~V}$. O acionamento da interface de controle foi executado por um programa desenvolvido em Quick Basic 4.5 (Microsoft, EUA).

\section{Reagente e soluções}

Todas as soluções foram preparadas a partir de reagentes de grau analítico (Merck ou Synth) e água destilada e desionizada.

Soluções contendo 0,$05 ; 0,1$ e $0,5 \mathrm{~mol} \mathrm{~L}^{-1}$ de $\mathrm{NaOH}$ foram preparadas dissolvendo-se quantidades apropriadas do respectivo hidróxido.

Solução estoque de Bromocresol púrpura (BCP) ( $\lambda_{\text {máx }}: 590 \mathrm{~nm}$ forma básica) foi preparada a partir da dissolução de $1 \mathrm{~g}$ do indicador em $5 \mathrm{~mL}$ de $\mathrm{NaOH} 0,1 \mathrm{~mol} \mathrm{~L}^{-1} \mathrm{e}$ diluída para $250 \mathrm{~mL}$ com água. A solução de trabalho foi preparada a partir de um volume de $5 \mathrm{~mL}$ da solução estoque, diluída para 1 L com água e algumas gotas da solução $0,05 \mathrm{~mol} \mathrm{~L}^{-1}$ de $\mathrm{NaOH}$, gotas suficientes para mudar a cor da solução do amarelo para o vermelho, $\mathrm{pH}$ próximo a 6,0 .

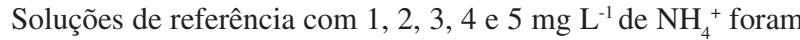
preparadas por diluições da solução estoque de $1000 \mathrm{mg} \mathrm{L}^{-1}$, preparada a partir da dissolução do sulfato de amônio.

\section{Instrumentos e procedimentos}

As análises foram realizadas empregando-se o sistema ASIA de análise em fluxo da Ismatec (Suíça) equipado com uma bomba peristáltica de 4 canais (IS 7610) com velocidade variável de 1 a 50 rpm, válvula eletrônica de injeção da amostra (IS 7630, Rheodyne, EUA), fotômetro de LED com comprimento de onda máximo em $605 \mathrm{~nm}$ (IS 7640 3EA) equipado com cela de fluxo de vidro, com volume interno de $180 \mu \mathrm{L}$ e percurso ótico de $10 \mathrm{~mm}$, câmara de difusão gasosa com percurso de $10 \mathrm{~cm}$ distribuídos na forma de ziguezague (sulcos de $1 \mathrm{~mm}$ ). A aspiração da amostra foi realizada por uma bomba peristáltica de rotação contínua (IS 7600), que preenchia uma bobina de amostragem de $250 \mu \mathrm{L}$ (tubos de polietileno com $0,8 \mathrm{~mm}$ d.i.).
O diagrama de fluxo do sistema FIA é apresentado na Figura 2. A amostra selecionada pelo autoamostrador é aspirada, preenchendo a alça de amostragem; após 30 s, a válvula injetora é acionada introduzindo a alíquota da amostra no percurso reacional. Por confluência a amostra contendo íons $\mathrm{NH}_{4}^{+}$recebe a solução de $\mathrm{NaOH} 0,5 \mathrm{~mol} \mathrm{~L}^{-1}$ produzindo $\mathrm{NH}_{3(\mathrm{~g})}$ que difunde através da câmara de difusão gasosa (membrana de polipropileno, tamanho de poro $0,1 \mu \mathrm{m}$, Millipore), sendo capturada na solução contendo bromocresol púrpura, $\mathrm{pH} \approx 6$. Em contato com a amônia o pH da solução é alterado mudando, consequentemente, a coloração do indicador, a qual é monitorada pelo detector fotométrico. A concentração analítica foi obtida correlacionando a área dos picos transientes e a concentração das soluções de referência. Esta sequência de amostragem, injeção e aquisição dos dados foi repetida para cada amostra, totalizando $117 \mathrm{~s}$ por amostra. Este tempo foi utilizado para sincronizar o autoamostrador com o sistema FIA, uma vez que os programas trabalhavam de forma independente.

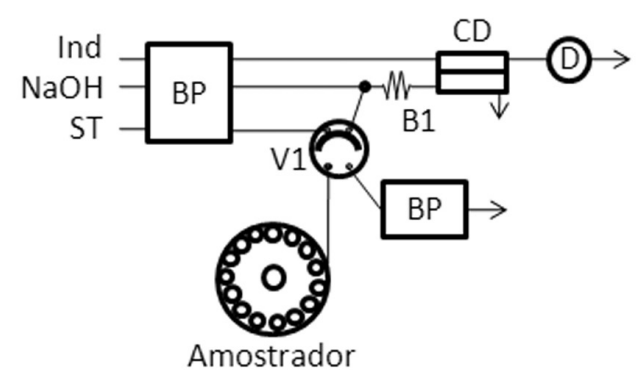

Figura 2. Diagrama de fluxos para a determinação de $\mathrm{NH}_{4}^{+}$. BP: Bomba peristáltica; V1: válvula Rheodyne; B1: bobina de mistura $(50 \mathrm{~cm}) ; C D$ : câmara de difusão gasosa; D: detector (605 nm); ST: solução transportadora, $\mathrm{H}_{2} \mathrm{O}$ (1,6 mL min $\left.{ }^{-1}\right)$; $\mathrm{NaOH}$ : solução 0,5 mol L-1 (0,7 mL min $\left.{ }^{-1}\right)$; Ind: solução de Bromocresol púrpura $20 \mathrm{mg} \mathrm{L}^{-1}\left(1,6 \mathrm{~mL} \mathrm{~min}^{-1}\right)$

A primeira etapa do programa de controle do autoamostrador inicia com testes dos motores de passo e a solicitação de posicionamento do capilar no frasco de amostragem inicial. Esta etapa torna-se necessária, uma vez que o autoamostrador não possui sensores de posicionamento. Na segunda etapa, ocorre a introdução do capilar no frasco da amostra e o início da contagem de tempo, este sincronizado ao início da operação do sistema FIA. Decorrido o tempo de um ciclo (117 s) o autoamostrador transfere o capilar para o frasco subsequente, permitindo a aspiração de outra amostra. Este ciclo é repetido por 50 vezes até a análise completa das amostras.

\section{RESULTADOS E DISCUSSÃO}

\section{Otimização do autoamostrador}

O primeiro parâmetro avaliado foi a sincronização do sistema FIA com o autoamostrador. Foi verificada uma defasagem de $2 \mathrm{~s}$ do autoamostrador em relação ao sistema FIA, após 90 min de operação (50 amostras). Entretanto, o tempo de $30 \mathrm{~s}$ estipulado para a amostragem foi suficiente para compensar essa defasagem, não apresentando nenhum tipo de problema.

Outro parâmetro avaliado foi o número de passos do motor 1 ou o giro em graus do braço de amostragem para a seleção do frasco. Os motores de passo utilizados são do tipo unipolar, no qual um giro de $360^{\circ}$ necessita de 100 passos. Os frascos das amostras foram posicionados a $22,5 \mathrm{~cm}$ do ponto central; com este raio foi possível posicionar 50 frascos de amostra com um ângulo de $7,2^{\circ}$ entre cada frasco. Para o posicionamento adequado do braço de amostragem, foram necessários dois passos completos deste motor. 
Uma das vantagens de se girar o braço de amostragem e não as amostras vêm do fato de que o peso movido pelo motor de passo é muito menor, não necessitando de motores com torque elevado. A desvantagem é que para se adicionar um número maior de amostras seria necessário ampliar o raio de posicionamento dos frascos, aumentando as dimensões do sistema ou reduzir o diâmetro dos frascos de amostra, diminuindo o volume de solução.

\section{Desempenho do autoamostrador}

O desempenho do autoamostrador foi avaliado empregando cinco soluções de referência de amônio $\left(\mathrm{NH}_{4}^{+}\right)$, com concentrações de 1 a $5 \mathrm{mg} \mathrm{L}^{-1}$ repetidas por dez vezes, sequencialmente. A Figura 3 mostra o registro dos sinais transientes destas soluções. Podemos observar que as 50 amostras foram corretamente amostradas em um período de aproximadamente $2 \mathrm{~h}$. A curva de calibração obtida pela área dos picos das dez replicatas das soluções de referências apresentou equação linear $Y=0,059( \pm 0,002) * X+0,005( \pm 0,002)$, com um ajuste de $\mathrm{R}^{2}=0,998$.

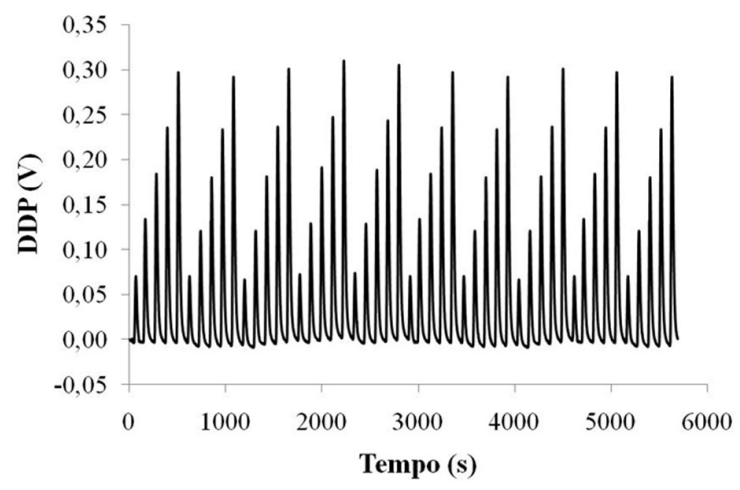

Figura 3. Sinais transientes de dez repetições das soluções de referência de 1, 2, 3, 4 e $5 \mathrm{mg} \mathrm{L}^{-1}$ de $\mathrm{NH}_{4}^{+}$

A passagem do capilar de amostragem de uma amostra para outra necessita de um tempo mínimo de $12 \mathrm{~s}$, portanto, sendo apropriado para sistemas em fluxo com frequência analítica menor que 90 amostras por hora. No sistema proposto, os frascos das amostras estão abertos e sujeitos a mudanças da concentração devido à evaporação do solvente ou a contaminações externas, entretanto, durante o período de operação estas perturbações não foram observadas. Quando necessário estes efeitos podem ser minimizados, reduzindo-se o orifício de entrada do capilar e controlando-se a temperatura ambiente.
A contaminação entre as amostras sequenciais não foi observada, entretanto, o uso de um capilar de PTFE (hidrofóbico) é apropriado para evitar que soluções concentradas possam interferir nas soluções mais diluídas. Etapas de limpeza do capilar também podem ser inseridas na programação, sendo apropriadas para amostras com maior viscosidade ou tendências ao acúmulo no capilar.

Como as unidades leitoras de disquete de $51 / 4$ polegadas estão em desuso comercial, motores de passo unipolar de outros equipamentos, como impressora jato de tinta ou matricial e digitalizadores de mesa, podem ser adaptados na montagem.

\section{CONCLUSÕES}

O autoamostrador mostrou-se adequado a aplicações em sistemas de análise por injeção em fluxo com comutadores mecanizados, sendo apropriado para análises de rotina que possuem um grande número de amostras, ou para sistemas que empregam reações químicas lentas, demandando vários minutos por amostras.

O custo, desconsiderando as unidades leitoras de disquete e o suporte de MDF, é de aproximadamente US\$25,00, gastos na aquisição dos componentes e confecção do circuito de controle dos motores de passo.

O emprego de sensores de posicionamento e circuitos de controle integrados ao sistema de detecção pode tornar o autoamostrador mais versátil e capaz de tomar decisões de operação a partir de parâmetros pré-estabelecidos.

\section{AGRADECIMENTOS}

Às agências de fomento FAPESP e CNPq pelos auxílios e bolsas concedidos. À UEMS pelo auxílio.

\section{REFERÊNCIAS}

1. Ruzicka, J.; Hansen, E. H.; Mosbœk, H.; Anal. Chim. Acta 1977, 92, 135.

2. Miranda, C. E. S.; Carrilho, E.; Gervasio, A. P.; Giné, M. F.; Quim. Nova 2002, 25, 412.

3. Ruzicka, J.; Hansen, E. H.; Flow Injection Analysis, $2^{\text {nd }}$ ed., Wiley: Nova York, 1988.

4. Pasquini C.; de Faria, L. C.; J. Automatic Chemistry 1991, 13, 143.

5. Figueredo, E. C.; Souza, L. R.; Magalhães, C. S.; Wisniewski, C.; Luccas, P. O.; Journal of Automated Methods and Management in Chemistry 2006, vol. 2006.

6. Ródenas-Torralba, E.; Rocha, F. R. P.; Reis, B. F.; Morales-Rubio, Á.; de la Guardiã, M.; Journal of Automated Methods and Management in Chemistry 2006, vol. 2006.

7. Messias, A. R.; http://www.rogercom.com.br, acessada em Julho 2008. 